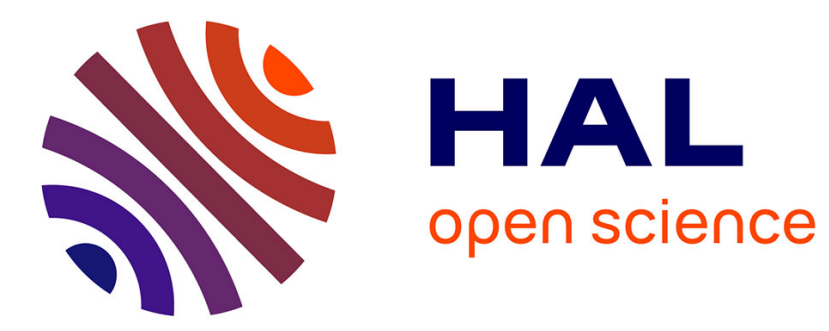

\title{
Sensitivity analysis of a fuzzy multiobjective scheduling problem
}

Sanja Petrovic, Carole Fayad, Dobrila Petrovic

\section{To cite this version:}

Sanja Petrovic, Carole Fayad, Dobrila Petrovic. Sensitivity analysis of a fuzzy multiobjective scheduling problem. International Journal of Production Research, 2008, 46 (12), pp.3327-3344. 10.1080/00207540601042472 . hal-00512954

\section{HAL Id: hal-00512954 https://hal.science/hal-00512954}

Submitted on 1 Sep 2010

HAL is a multi-disciplinary open access archive for the deposit and dissemination of scientific research documents, whether they are published or not. The documents may come from teaching and research institutions in France or abroad, or from public or private research centers.
L'archive ouverte pluridisciplinaire HAL, est destinée au dépôt et à la diffusion de documents scientifiques de niveau recherche, publiés ou non, émanant des établissements d'enseignement et de recherche français ou étrangers, des laboratoires publics ou privés. 


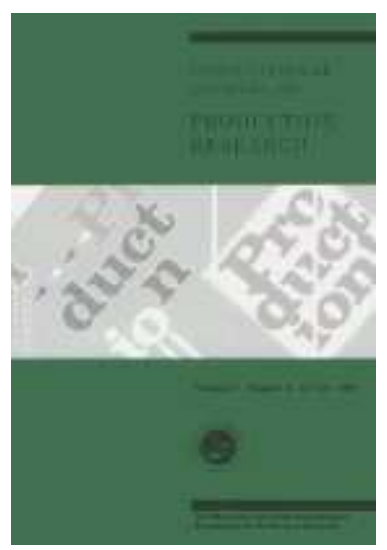

\section{Sensitivity analysis of a fuzzy multiobjective scheduling problem}

\begin{tabular}{|r|l|}
\hline Journal: & International Journal of Production Research \\
\hline Manuscript ID: & TPRS-2006-IJPR-0346.R1 \\
\hline Manuscript Type: & Original Manuscript \\
\hline Author: & $26-$ Sep-2006 \\
\hline & $\begin{array}{l}\text { Complete List of Authors: } \\
\text { Petrovic, Sanja; University of Nottingham, School of Computer } \\
\text { Science and IT University of Nottingham, School of Computer } \\
\text { Information Sciences }\end{array}$ \\
\hline Keywords: & $\begin{array}{l}\text { GENETIC ALGORITHMS, MULTI-CRITERIA DECISION MAKING, } \\
\text { SCHEDULING, JOB SHOP SCHEDULING }\end{array}$ \\
\hline Keywords (user): & SENSITIVITY ANALYSIS, FUZZY SETS \\
\hline
\end{tabular}

\section{scholarONE" \\ Manuscript Central}




\section{Sensitivity analysis of a fuzzy multiobjective scheduling problem Sanja Petrovic", Carole Fayad", Dobrila Petrovic ${ }^{+}$}

"University of Nottingham, School of computer Science and IT, UK, \{sxp I cxf@cs.nott.ac.uk\} ${ }^{+}$Coventry University, Faculty of Engineering and Computing

D.Petrovic@coventry.ac.uk

Deleted: School of Mathematical and Information Sciences
Abstract. This paper concerns sensitivity analysis of a class of complex job shop scheduling problems which are characterized by: (1) a large number of jobs and machines, (2) uncertain jobs processing times, and (3) multiple measures of schedule performance including average weighted tardiness, the number of tardy jobs, the total setup times, the total idle time of machines, and the total flow times of jobs. The base schedule is generated by applying a new fuzzy multiobjective genetic algorithm which takes into consideration batching of the jobs of a similar type, jobs' lots sizing and load balancing of the machines. The aim of the proposed sensitivity analysis of a generated schedule is to investigate the consequences of prolongations of job processing times on the measures of schedule performance, The processing times are described by triangular fuzzy numbers and their prolongation is done by expanding the supports of fuzzy numbers. The sensitivity analysis is performed through a series of numerical experiments. The effects of prolongations of job processing times on the measures of performance of a generated schedule are recorded and analysed. It is shown that the sensitivity analysis is among the primaries in evaluating the quality of a generated schedule. The sensitivity analysis is used in identifying the critical jobs and the critical machines which have the properties that the prolongations of their processing times produce the largest deteriorations of the performance measures and the overall quality of a generated schedule.

Keywords: sensitivity analysis, production scheduling, job shop scheduling, fuzzy sets, multiobjective decision making, genetic algorithm
Deleted: taking

Deleted: changes of basic model parameters, such as processing times of jobs.

Deleted: imprecisely specified and modelled

Deleted: The change of each job processing time is done by expanding the support of the corresponding fuzzy number, in such a way that the lower bound remains fixed and the upper bound increases in discrete steps.

Deleted: such changes

Deleted: all of

Deleted: essential

Deleted: Besides, it helps in determining

Deleted: with respect to measures of performance and to the overall quality of a schedule. 
1. Introduction

A typical production scheduling problem concerns the allocation of resources (e.g. equipment, materials, labour, etc.) to carry out tasks over time under some constraints. Various machine configurations that exist in real-world production/manufacturing environments have been studied in the literature (Leung, 2004, Pinedo, 2002), starting from the simplest case of a single machine, through a flow shop which includes multiple machines in a series, to the most complex job shop environments (Blazewicz et al. 1996).

Production/manufacturing processes are typically described by various temporal relations, resource requirements and capacity constraints, which involve a wide range of parameters, such as job release dates, processing times, due dates, priorities of jobs, machine setup times, etc. One can find a large number of production scheduling models and algorithms reported in the literature, which assume that all model parameters are well-known and precisely determined. However, underlying almost all real production systems are activities fraught with uncertainties and imprecisions. For example, the duration of tasks involving humans is seldom known precisely or due-dates may not always be quite as rigid as they are supposed to be in the classical scheduling models. Further, the machines are not absolutely reliable and sometimes the failures of the machines occur with random durations. This often prevents the results of deterministic scheduling theory from being applied in practice. As a natural step ahead, the deterministic scheduling models and algorithms have been extended to tackle stochastic parameters that assume that processing times are stochastic by nature with specified probability distributions (Dempster et al. 1982). However, the probabilistic characteristics of parameters in scheduling models are often lacking in practice. Statistics about the duration of processes/jobs can be both imprecise and/or incomplete, or sometimes do not even exist. In these cases, the application of the traditional approaches in treating uncertainty in the processing times of operations based on probability theory becomes difficult and often inappropriate.

One approach to treating uncertainties in the durations of processing times that are always present in real-world scheduling is based on concepts of fuzzy sets theory. Fuzzy scheduling models have been developed and published in the last decade,
Deleted: at

Deleted: 
mainly using fuzzy sets in different ways (Slowinski and Hapke, 2000). We identified three important domains in scheduling problems where fuzzy sets have been applied.

(1) Imprecise scheduling parameters, such as processing times and due dates, have been represented as fuzzy numbers and operations on them have involved fuzzy arithmetic (Sakawa and Kubota, 2000).

(2) Fuzzy dispatching rules have been developed, where linguistic qualifiers, modelled by fuzzy sets, have been used to express the concepts of similarity or closeness. For example, fuzzy versions of well known dispatching rules such as SPT (shortest processing time) and slack time rules have involved fuzzy qualifiers such as short, small, much longer than, etc. (Grabot and Geneste, 1994)

(3) Fuzzy sets have been used to represent flexible constraints, usually of temporal nature, such as release dates or due dates, whose violation has to be minimised, and the best schedule requests the least relaxation of these constraints (Fagier, 1996).

This paper concerns a class of production scheduling problems, and, in particular, sensitivity analysis of generated schedules with respect to changes of processing times of job operations. Our research is focused on a job shop problem with parallel machines in a printing company Sherwood Press in Nottingham, UK. This scheduling problem is characterised by: (a) a large number of jobs, (b) uncertainty of processing times of job operations and their due dates, and (c) multiple measures that are used to evaluate the performance of the generated schedules. In the first phase of our research, a new fuzzy multicriteria genetic algorithm for scheduling (FMGAS) has been developed (Petrovic et al. 2005, Fayad and Petrovic, 2005, Petrovic and Fayad, 2005). The printing company wanted to get some additional characteristics of the generated schedules, in particular what the effects of prolongations of the operation processing times are. This is, of course, a very common case in the practice. In order to obtain confidence in the schedules generated by FMGAS, sensitivity analysis is performed to test the consequences of changes of the processing times of jobs. The aim of this paper is to present the results of the sensitivity analysis in a real world scheduling problem.

The literature on sensitivity analysis of scheduling problems is rather limited. Sotskov (1991) investigated the so-called stability radius for a schedule that is optimal with respect to a single criterion, when the processing times of jobs' operations were
Deleted: and 
changed. It was concluded that the proposed methods were of a high complexity, apart from some special cases of scheduling problems which were tractable. James and Buchanan (1998) examined experimentally the sensitivity of a schedule to the earliness and tardiness penalties in the objective function. Kolen et al. (1994) investigated sensitivity of some dispatching rules to changes of the operation processing times in a parallel machine environment. Hall and Posner (2004) provided a systematic study of issues that arise in the sensitivity analysis in scheduling problems. They investigated how to obtain a new optimal schedule for a scheduling problem with modified parameters by using the optimal schedule for the original problem.

We designed a series of numerical experiments to perform sensitivity analysis in the scheduling problem under consideration. Numerical experiments were used due to the fact that any analytical approach to the sensitivity analysis appears to be too complex. It is practically impossible to derive analytical formulae which would describe the effects of changing operation processing times on the measures of performance of the generated schedule. The sensitivity analysis will help in getting a better picture of the generated schedules in the cases of unexpected prolongations of operation processing times. In addition, while in the majority of other studies of sensitivity analysis of schedules, a single objective has been considered, in this research we take into account multiple objectives simultaneously ${ }_{\uparrow}$ This makes the sensitivity analysis even more complex. Apart from investigating the effects of changing operation processing times on the measures of performance of the schedule, our numerical experiments enable us, also, to identify some critical jobs and some critical machines on the shop floor, which are critical with respect to the different measures of performance of the generated schedule.

The paper is organised as follows. Section 2 gives a scheduling problem statement related to a real-world example from the printing industry. That was the main motivation to develop a new procedure for a sensitivity analysis in scheduling problems. Section 3 gives a concise overview of the genetic algorithm FMGAS that was developed to solve the given scheduling problem. In Section 4, the sensitivity analysis is described as essential in evaluating the overall quality of the obtained schedule. The attention is focussed to identify critical jobs and critical machines. It is followed by the conclusions and some views on the future work, given in Section 5 .

Deleted: at

Deleted: of
Deleted: , and i Deleted: jobs

Deleted: a
Deleted:

Deleted: jobs' 
2. Fuzzy multiobjective scheduling problem

The notation used in the problem statement and throughout the paper is as follows:

\begin{tabular}{|c|c|c|}
\hline$N$ & total number of jobs & \\
\hline$J_{j}$ & job, $j=1, \ldots, N$ & \\
\hline$M$ & total number of machines & \\
\hline$M_{i}$ & machine, $i=1, \ldots, M$ & \\
\hline \multirow[t]{2}{*}{$(i, j)$} & operation of job $J_{j}$ processed on machine $M i \quad i=1, \ldots, M$, & Deleted: $M_{i}$ \\
\hline & $j=1, \ldots, N$ & Deleted: \\
\hline$\tilde{p}_{i j}$ & fuzzy processing time of operation $(i, j)$ & Deleted: $p_{i j}$ \\
\hline \multirow[t]{2}{*}{$r_{j}$} & release date of job $J_{j}, j=1, \ldots, N$, which denotes the time when & \\
\hline & the job can start its processing & Deleted: \\
\hline$\tilde{d}_{j}$ & 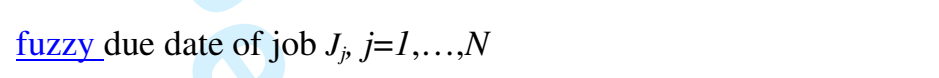 & Deleted: $d_{j}$ \\
\hline$\tilde{C}_{j}$ & fuzzy completion date of job $J_{j}, j=1, \ldots, N$ & Deleted: $C_{j}$ \\
\hline \multirow{2}{*}{$\tilde{T}_{j}$} & \multirow{2}{*}{ fuzzy tardiness of job $J_{j}, j=1, \ldots, N$, } & Deleted: $T_{j}$ \\
\hline & & Deleted: $T_{j}=\max \left(C_{j}-d_{j}, 0\right)$ \\
\hline$w_{j}$ & 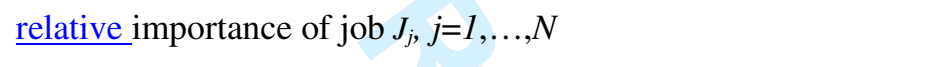 & \\
\hline$F$ & total number of families of jobs & \\
\hline$F_{f}$ & family of jobs, $f_{-}=1, \ldots, F$ & \\
\hline$a$ & time needed for setup of a machine when different families of & \\
\hline & jobs are processed one after the other & \\
\hline$\Theta$ & planning horizon & \\
\hline \multirow[t]{2}{*}{$T$} & total number of discretized time periods of length $\Delta$ in the & \\
\hline & planning horizon $\Theta$ & Deleted: \\
\hline . & & Deleted: \\
\hline$t$ & discrete period in the planning horizon, $t_{-}=1, \ldots, T$ & \\
\hline$Q_{1}$ & average weighted tardiness & \\
\hline$Q_{2}$ & number of tardy jobs & \\
\hline$Q_{3}$ & total setup time & \\
\hline$Q_{4}$ & total idle time of machines & \\
\hline$Q_{5}$ & total flow time & \\
\hline
\end{tabular}


$S_{k}$

satisfaction grades of the corresponding objectives $Q_{k_{2}}$ $\underline{\mathrm{k}=1, \ldots 5}$

$\Phi$ overall quality of a schedule
Deleted: $, k=1, . ., 5$

\section{Deleted: the}

Deleted: ,

Deleted: $\Phi=\left(S_{1}+S_{2}+S_{3}+S_{4}+S_{5}\right) / 5$

\subsection{Problem statement}

The job shop scheduling problem considered in this research consists of $N$ jobs $J_{l}, \ldots, J_{j}, \ldots, J_{N}$, which have to be scheduled on $M$ machines $M_{l}, \ldots, M_{i}, \ldots, M_{M}$. Machines are grouped into working centres. A working centre consists of machines; among them some machines are identical. To each job $J_{j 2}$ a release date $r_{j}$ and a due date $\tilde{d}_{j}$ are assigned, which denote the date when the job can start its processing, and the date when the job is due to finish its processing, respectively. It is assumed that the due dates of the jobs are imprecise. The production manager can express his/her dissatisfaction with the tardiness of the jobs that cannot meet their due dates.

The processing of job $J_{j}$ on machine $M_{i}$ is referred to as an operation, represented by an ordered pair $(i, j)$. Operation processing times are uncertain and modelled by fuzzy sets denoted by $\tilde{p}_{i j}$. Precedence constraints are imposed on the order of operation processing. A weighting coefficient $w_{j}, j=1, \ldots, N$, is assigned to each job $J_{j_{k}}$ It determines the importance of the job from the point of view of the manager and is subjective in nature.

Jobs are grouped into $F$ families. Jobs which belong to the same family do not

Deleted: identical and non-identical

Deleted: Identical machines are those machines whose characteristics are the same.

Deleted:

Deleted:

Deleted: $d_{j}$

Deleted: and they allow $\mathrm{t}$

Deleted:

Deleted: to

Deleted:

Deleted: , with the processing time denoted by $p_{i j}$. Precedence constraints are imposed on the order of operation processing.

Deleted:

Deleted:

Deleted: which

Deleted: $f, f=1, \ldots, F$,

Deleted: a
Deleted: a
Deleted: so
Deleted: The
are
Deleted: $\theta$

The scheduling problem is formulated as follows. Find a non-preemptive sequence of operations of $N$ jobs on $M$ machines subject to the following constraints: (1) the precedence constraints which ensure that the processing sequence of 
operations conforms to the predefined order, and (2) the capacity constraints which ensure that a machine processes one job at a time.

The performance of the schedule is measured using the following five measures of performance:

$Q_{1}$ : the average weighted tardiness of schedule $s$

$$
Q_{1}(s)=\frac{1}{N} \sum_{j=1}^{N} w_{j} \tilde{T}_{j}
$$

where $\tilde{T}_{j}$ is the fuzzy tardiness of job $J_{j}$, which takes into account the difference between the completion and due date of the job. Due to the fuzzy processing times of job operations, the completion time and tardiness of the job are also fuzzy.

$Q_{2}$ : the number of tardy jobs in schedule $s$

$$
Q_{2}(s)=\sum_{j=1}^{N} u_{j}, \text { where } u_{j}=\left\{\begin{array}{cc}
1 & \text { if } j o b J_{j} \text { is tardy } \\
0 & \text { otherwise }
\end{array}\right.
$$

$$
Q_{3}(s)=\sum_{t=1}^{T} \sum_{f=1}^{F} a X_{f t}
$$

where $X_{f t}= \begin{cases}1 & \text { if there is a need for a setup for family } f \text { of jobs at period } t \\ 0 & \text { otherwise }\end{cases}$

$X_{f t}$ is referred to as the changeover coefficient and parameter $a$ denotes the time needed to make a machine ready for a changeover of the job families.

$Q_{4}$ : the total idle time of the machines in schedule $s$, where the idle time is defined as the time within the planning horizon $\Theta$ during which the machines are used neither for processing of jobs nor for the setup of the machine:

$$
Q_{4}(s)=\sum_{i=1}^{M}\left(\left(\tilde{C}_{i a}-\tilde{s}_{i b}\right)-\sum_{j=1}^{N} \tilde{p}_{i j}\right)-\sum_{t=1}^{T} \sum_{f=1}^{F} a X_{f t}
$$

where $\tilde{C}_{i a i a}$ and $\tilde{s}_{i a} s_{i b}$ are the fuzzy completion time of the last operation and the fuzzy start time of the first operation on machine $M_{i}$ within the planning horizon $\Theta$,

Deleted: $T_{j}=\max \left(C_{j}-d_{j}, 0\right)$ Deleted: is the tardiness of job $J_{j}$, while $d_{j}$ and $C_{j}$ are the due date and the completion time of the job, respectively Deleted:

Deleted: 
In this section a scheduling problem in the printing company Sherwood Press is presented. This job shop problem involves 18 machines, which are grouped into 7 work centres: Printing, Cutting, Folding, Card-inserting, Embossing and Debossing, Gathering and Finishing (includes machines for stitching, trimming and packaging). All five measures of performance have to be maximised.

\subsection{A real-world scheduling problem}

Each job has a predetermined order of processing on machines in some or in all work centres. Jobs are grouped into families on the basis of their colour requirements. Printing machines require setup time when the jobs of different families, which require different colours, are scheduled for printing one after the other. It is assumed that the time needed for the setup of all the printing machines is 20 minutes $(a=20)$.

The production manager estimates the processing time of each operation, taking into account the nature of the machine in use. Some machines are automated and can be operated at different speeds, while others are staff-operated and, therefore, the processing times are staff-dependent. Uncertain processing time of operation $(i, j)$ is modelled by a fuzzy set $\tilde{p}_{i j}$ with a triangular membership function given by a triplet $\left(p_{i j}^{1}, p_{i j}^{2}, p_{i j}^{3}\right)$, where $p_{i j}^{2}$ identifies a typical value of the processing time, usually called modal point (Klir, 1998), whereas $p_{i j}^{1}$ and $p_{i j}^{3}$ are the lower and the upper bounds of the processing time. They reflect the spread of the possible processing times specified imprecisely as 'about a given value', and in this model they are set to be equal to $0.9 \cdot p_{i j}^{2}$, and $1.1 \cdot p_{i j}^{2}$, respectively. An example of a membership function

Deleted: (this point is Deleted: ) for an uncertain processing time is shown in Figure 1. 
Deleted: " Deleted: “

Following the scheduling practice in Sherwood Press, we classify jobs into three groups on the basis of their priorities and the corresponding tolerance of their tardiness: high, medium and low priority jobs. The group that job $J_{j}$ is classified into determines its weighting coefficient $w_{j}$. Jobs of high priority (Priority 1 ) are expected to be processed in time, with no delay, and therefore, crisp due dates are defined for Deleted: there are them. Jobs of medium priority (Priority 2) have due dates that can be extended by up to two additional days, whereas jobs of low priority (Priority 3) are given a delay tolerance of up to one week on top of the originally set due dates. Jobs of Priority 1 , Priority 2 and Priority 3 are assigned weighting coefficients $0.7,0.4$, and 0.3 , respectively. The imprecise due dates $\tilde{d}_{j}, j=1, \ldots, N$, are described by a trapezoidal membership function, as illustrated in Figure 2. Membership degrees represent dates. 
Figure 2. Membership functions of three fuzzy sets Due date of job $J_{j}$ if the job has

Priority 1, Priority 2 and Priority 3

\section{A fuzzy multiobjective genetic algorithm for scheduling}

A fuzzy multiobjective genetic algorithm for scheduling (FMGAS) which consist of three phases was developed for generating schedules for the described scheduling problem. Each of the three phases is briefly explained below.

Phase 1. Lots sizing: For each job a decision has to be made whether to split it into lots and, if it is the case, the size of lots has to be determined. A fuzzy IF-THEN rule-based system was developed which drew the conclusion whether to split-up jobs and determined the size of each lot using four linguistic variables: size of the job, Deleted: draws slack of the job, workload on the shop floor, and the priority of the job. (Petrovic et al. Deleted: s 2005). In the remainder of the paper we will refer to the lots as jobs. Splitting a job into lots leads to an increased number of jobs, because each lot is considered as a job. Consequently, there is an increase in the total number of operations to be scheduled for processing on the machines. The created lots have the same characteristics as the job they originated from, for example release time, due date, etc.

Phase 2. Machine allocation: A machine is allocated to each operation of each job. A genetic algorithm was developed to balance the load of the identical machines in each work centre (Petrovic and Fayad, 2005). 
Phase 3. Sequencing of jobs on the machines: a fuzzy multi-objective genetic algorithm was developed for sequencing of jobs on the machines (Fayad and Petrovic, 2005). It evolves the decision on which dispatching rules to use for scheduling of jobs' operations on each machine taking into consideration both imprecise processing times and due dates of jobs, and the determined sizes of lots. The dispatching rules are: Early Due Date (EDD), Shortest Processing Time (SPT), Longest Processing Time (LPT), Most Work Remaining (MWR), Same Family Together (SFT), and Highest Priority (HP). The following characteristics of the jobs are used in the ranking of their operations: $E D D$ uses the due date of the jobs, $S P T$ and $L P T$ employ the required processing times of the jobs, $M W R$ calculates the required processing time for all the remaining operations of the jobs, SFT takes into account the family of jobs that the operation belongs to, i.e. the priority is given to operations of the same family as the previously allocated operation, $H P$ is based on the priority of the jobs. These rules operate in the following way: if there is only one operation ready for processing, it will be processed immediately; if there are more than one operations available, the dispatching rule associated with the machine will determine the order of their processing.

In order to calculate the completion time of each job, fuzzy operation processing times have to be summed up. The standard fuzzy addition based on the extension principle, which preserves the triangular membership function of the resulting completion time, is used (Dubois and Prade, 1978). The obtained measures of schedule performance that take into consideration fuzzy processing times and fuzzy completion times are also fuzzy numbers. The satisfaction grades are introduced for each measure of performance to reflect the production manager's satisfaction with the achieved values. Satisfaction grades take values from the $[0,1]$ interval, where 0 represents full dissatisfaction, and 1 represents full satisfaction with the achieved value of the measure of performance. The calculation of the satisfaction grade of each measure of performance is briefly explained here, while more details can be found in (Petrovic et al al. 2005).

$\underline{S}_{1}$ : The satisfaction grade of the average weighted tardiness is calculated as the average of the satisfaction grades of the tardiness of all the jobs. The satisfaction grade of job's tardiness is determined taking into consideration the intersection of the job's fuzzy completion time and the fuzzy due date.

\begin{tabular}{l|}
\hline Deleted: preferences to \\
\hline Deleted: $[0,1]$ \\
\hline Deleted: a \\
\hline Deleted: $S_{1}$ : For each job the \\
satisfaction grade of its tardiness is \\
calculated taking into account the \\
intersection of the job's fuzzy completion \\
time and the fuzzy due date. The \\
satisfaction grade of the average weighted \\
tardiness is calculated as the average of \\
the satisfaction grades of the tardiness of \\
all the jobs. II \\
$S_{2}:$ A threshold of the satisfaction grade \\
of the job tardiness is introduced to \\
determine whether a job is tardy. A linear \\
function is used to map the number of \\
tardy jobs in a schedule into a satisfaction \\
grade of the number of tardy jobs. II \\
$S_{3}, S_{4}$ and $S_{5}:$ Fuzzy values of these \\
measures of performance are defuzzified \\
using the centre of gravity. These values \\
are mapped into satisfaction grades using \\
linear functions. \\
Deleted: \\
\hline
\end{tabular}


$\underline{S}_{2}:$ Firstly, in order to determine whether a job is tardy or not, a threshold of $\underline{\text { the corresponding satisfaction grade is introduced; if the satisfaction grade of job's }}$ tardiness is greater than the threshold, the job is tardy; otherwise, it is not. Secondly, a linear function is used to map the number of tardy jobs in a schedule into the corresponding satisfaction grade of the number of tardy jobs. $S_{3}$ : The maximum and minimum setup times are mapped into satisfaction grades 0 and 1 , respectively, while other values of setup times (between maximum and minimum values) are mapped into linearly increase.

$\underline{S}_{4}$ and $S_{5}$ : Due to fuzziness of the operation processing times, the total idle $\underline{\text { time of machines and the total flow time become fuzzy too. These fuzzy values are }}$ defuzzified first using the centre of gravity method, and, then mapped into the corresponding satisfaction grade scale, using linear functions.

The parameters of the linear functions used in the calculation of satisfaction grades reflect the scheduling policy of the company. For example, when assessing $Q_{2}$ the production manager is fully dissatisfied with a schedule with over $15 \%$ of the tardy jobs. Consequently, a liner function is defined to determine the satisfaction grade, where the satisfaction grade linearly decreases from 1, (when there is no tardy job, i.e. the percentage of tardy jobs is 0 ), to 0 , when the percentage of tardy jobs is greater or equal to 15 .

In order to evaluate the overall quality of a schedule, the fitness function $\Phi$ is defined as the average of the five satisfaction grades:

$$
\Phi=\left(S_{1}+S_{2}+S_{3}+S_{4}+S_{5}\right) / 5
$$

The larger the $\Phi(s)$, the better the schedule s.
Deleted: greated

Deleted: tardy;otherwise

Deleted: II
Deleted: An average of the five satisfaction grades of all measures of performance is calculated. It is used as the fitness function $\Phi$ to evaluate the overall quality of schedules.II 


\section{Sensitivity analysis}

Data sets obtained from Sherwood Press are used to carry out a series of experiments. The input data for each job are: the identification number of the job, the order of machines on which the job requires processing, the fuzzy processing time of job operations on each of the machines, the required quantity of the items, the family of the job, the release date, the due date and the priority of the job. The planning horizon is 4 weeks, discretized in minute steps. The workload of the shop floor is calculated taking into consideration the required printing orders. Initial number of jobs is 158 . After splitting jobs into lots the total number of jobs increases to 298, which consist of 1044 operations.

The aim of the sensitivity analysis in scheduling is to test the consequences of changes of the basic input parameters, i.e. processing times of the job operations. Data from one month that is considered to be rather busy were chosen to illustrate the results of the sensitivity analysis. The sensitivity analysis is performed by numerical experiments, which take as input the base schedule generated by the FMGAS. The change of an operation processing time in each experiment is done by expanding the support of the corresponding fuzzy set, where the support includes all processing times with membership degrees greater than 0 . The support of the fuzzy processing time is expanded in such a way that the lower bound remains fixed, while the upper bound increases gradually, with the increment of $5 \%$ of its modal value, up to the double of the modal value. Increasing the upper bound of the processing time means that the value of the processing time has become increasingly pessimistic. The changes of the processing times are made sequentially, job by job. The effects of such changes on each individual measure of performance, on the corresponding satisfaction grades and on the fitness function, i.e., the overall quality of the schedule are recorded.

The schedule produced by applying the FMGAS will be referred to as the base schedule. The performance of the base schedule measured by five measures of performance and the corresponding satisfaction grades are given in Table 1. The obtained values of the measures of performance, except $Q_{2}$ are fuzzy values The crisp $_{2}$ values in Table 1 are produced by defuzzification, using the centre of gravity method.

Deleted: $\left(Q_{l}\right.$ Deleted: , $\left.Q_{3}-Q_{5}\right)$

Deleted: due to the fuzziness of processing times of operations.

Deleted: and they are shown in Table 1 . 
Table 1. Measures of performance and the corresponding satisfaction grades of the base schedule

\begin{tabular}{|l|l|l|}
\hline Measure of performance & Value & Satisfaction Grade \\
\hline Average weighted tardiness $Q_{1}$ & 789 minutes & 0.435 \\
\hline Number of tardy jobs $Q_{2}$ & 9 jobs & 0.713 \\
\hline Total setup time $Q_{3}$ & 722 minutes & 0.308 \\
\hline Total idle time $Q_{4}$ & 189370 minutes & 0.442 \\
\hline Total flow time $Q_{5}$ & 460602 minutes & 0.838 \\
\hline Fitness function $\Phi=0.547$ & & \\
\hline
\end{tabular}

In the numerical experiments that were carried out, we notify a change of a measure of performance if its satisfaction grade is changed by at least $3 \%$ of the corresponding value in the base schedule. Value of $3 \%$ is chosen because it corresponds to the decrease of the satisfaction grade of $Q_{2}$ if one more tardy job appears in the new schedule compared to the base schedule. This value is used for all five measures of performance in order to treat all of them equally. Of course, the value is highly subjective, and reflects the preference of the production manager.

An important question that arises when a processing time of an operation is prolonged is what changes of the base schedule are useful to examine. A number of characteristics are important for the base schedule, such as the start time of the processing of each operation, the operation sequence on each machine, a dispatching rule associated with each machine, which determines the operation sequence on that Deleted: a pessimistic Deleted: job is increased Deleted: a

Deleted: machine, and the measures of performance of the schedule. Every increase of the processing time of an operation leads inevitably to the corresponding increase of the start times of the later operations. Therefore, the start times of the operations will not be used as the indicator of the change of the base schedule. It is more meaningful to evaluate the change of measures of performance, and consequently, the change of the overall quality of the new schedule.

Once the operation(s) processing time on a machine is prolonged we can keep the same operation sequence on each machine as it was in the base schedule and Deleted: prolongedchanged we calculate the new measures of performance. However, we find that it is more interesting to keep the dispatching rules associated with the machines in the base schedule fixed. Then, due to the change of operation processing times on the machine,

Deleted: D Deleted: thea machine 
the associated dispatching rule can change the operations sequence on that machine. It can also affect the sequence on some other machines to which the dispatching rule Most Work Remaining is assigned. Four numerical experiments are designed and performed which help the production manager to evaluate the quality of a schedule with respect to all the measures of performance from different aspects. In the following, the selected results of the sensitivity analysis in the scheduling problem which rises in the printing company are presented.

Deleted: We examined the impact that a change of the operation sequence on a machine could have on the performance of the base schedule.

Deleted: were

Deleted: raises

4.1. Effects of the change of an operation processing time on the measures of performance

The upper bound of the processing time of each operation is changed, one by one, and the change of each measure of performance is recorded. The pseudo-code of the algorithm used is given in Figure 3.

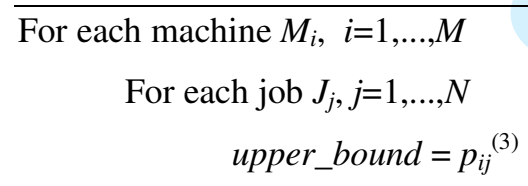

Figure 3. The pseudo-code of the algorithm for recording the measures of performance and satisfaction grades with increasing processing time of each operation

The numerical experiments show that changes of operation processing times produce some irregular effects on the performance measure of the base schedule. Such phenomena are expected due to the high complexity of the scheduling problem, and also, due to the fact that the scheduling problem is stated and solved by applying discrete mathematical structures. In our experiments, we record all five measures of 
performance and the results show that they are partially conflicting: some among the measures of performance have been improved, while some of them have deteriorated as a consequence of changes of an operation processing time. Irregularities are complete in the sense that two possible situations occur: (1) starting from a certain prolongation of the operation processing time there is either improvement or deterioration of the performance values, (2) under some prolongations of the operation processing time the considered measure of performance improves, while under the other prolongations, it deteriorates. For example, Figure 4 shows that with the change of the upper bound of the processing time of operation $(3,1), Q_{4}$ (the total idle time) increases and $Q_{5}$ (the total flow time) decreases, While $Q_{4}$ starts to deteriorate when the upper bound of the processing time of the operation is greater than $50 \%$ of its modal value, $Q_{5}$ improves $_{\mathrm{v}}$

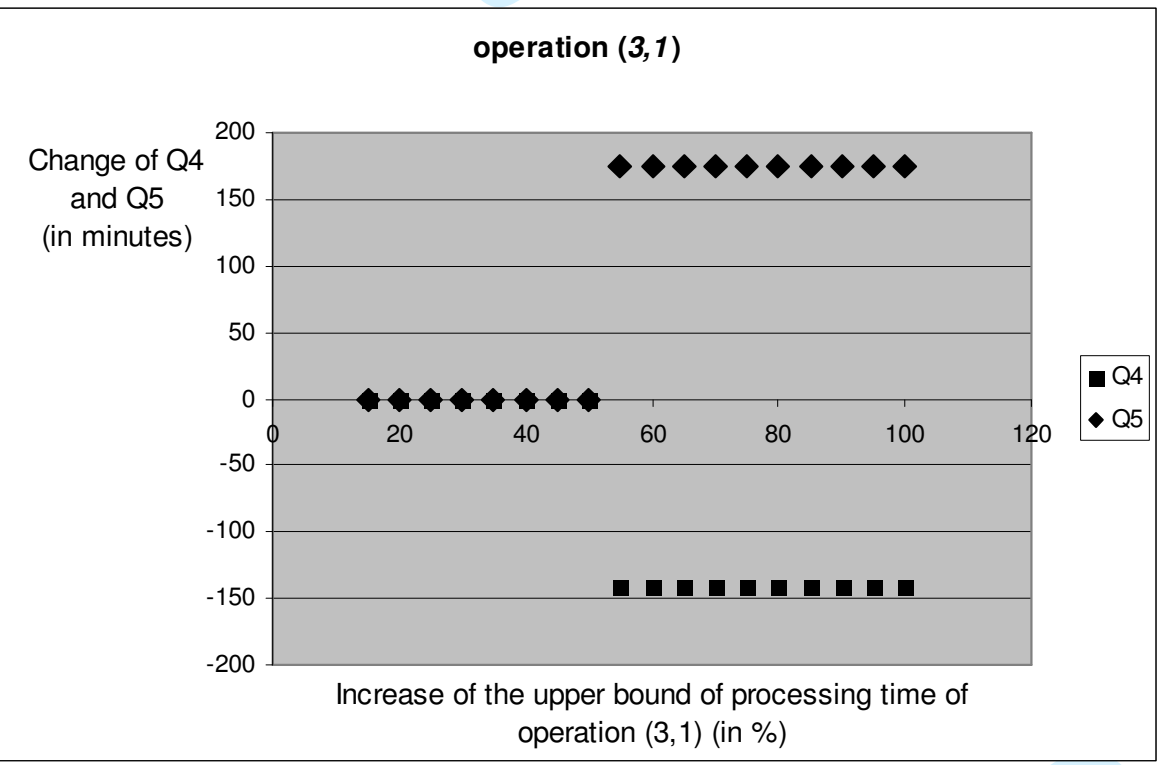

Figure 4. The effect of changing the upper bound of the processing time of operation $(3,1)$ on $Q_{4}$ (the total idle time) and $Q_{5}$ (the total flow time)

The improvement of a performance measure occurs due to the change of the sequence of jobs on the machine on which prolongation of the operation processing time occurred. This can even lead to the change of operation sequence on the other

Deleted: them

\begin{tabular}{l} 
Deleted: all \\
Deleted: change \\
Deleted: changes \\
Deleted: some \\
\hline Deleted: s \\
\hline Deleted: they \\
\hline Deleted: and decrease of the \\
\hline $\begin{array}{l}\text { Deleted: } \\
\text { total flow time }\left(Q_{5}\right), \text { with the change of } \\
\text { the upper bound of the processing time of } \\
\text { operation }(3,1)\end{array}$ \\
\hline Deleted: \\
Deleted: \\
\hline
\end{tabular}

Deleted: the total idle time ( Deleted: )

Deleted:

Deleted: and the total flow time ( Deleted: )

Deleted: the 
machines. The change of the sequence(s) can lead to the improvement of the performance of the schedule.

On the other hand, Figure 5 shows that the changes of a measure of performance can be quite irregular. It illustrates the changes of total setup time caused by prolongation of $p_{1}, 16$. Figure 6 shows that it can happen that only a specific prolongation of the operation processing time of the particular job causes changes of \begin{tabular}{|l|}
\hline Deleted: $F$ \\
\hline Deleted: the \\
\hline Deleted: $s$ \\
\hline $\begin{array}{l}\text { Deleted: increased processing time of } \\
\text { operation }(1,16)\end{array}$ \\
Deleted: single change \\
\hline
\end{tabular} the schedule performance. These irregularities contribute to the conclusion that only numerical experiments can help in getting a better picture about the sensitivity of the performances of the base schedule to the prolongation of an operation processing time.

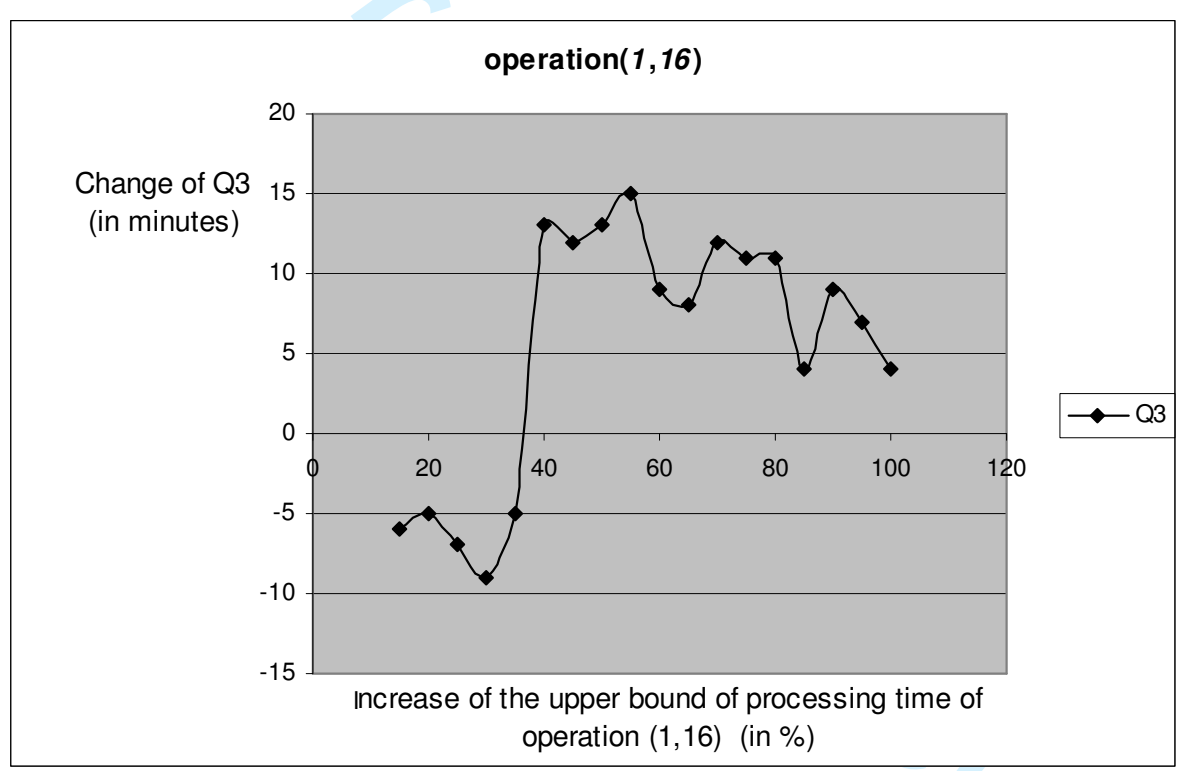

Figure 5. The effect of changing the upper bound of the processing time of operation $(1,16)$ on $Q_{3}$ (the total setup time)
Deleted: the total setup time ( Deleted: 
Figure 6. The effect of changing the upper bound of the processing time of operation $(136,2)$ on $Q_{4}$ (the total idle time), and $Q_{5}$ (the total flow time)

4.2 Critical operations

For the production manager it is useful to identify operations from which the prolongations of processing times have particularly high influence on the measures of performance of the schedule. Such operations will be called critical. An experiment is designed to identify critical operations.

In this experiment prolongation of the processing time of each operation is performed as in the previous experiment, but this time the range of changes of each of the performance measures, the corresponding satisfaction grades, and the overall quality of the schedules are recorded. Such an analysis enables the identification of the operation whose prolongation of its processing time affects the most the overall quality of the schedule or a single measure of performance. It also enables the evaluation of the range of possible changes of the performance of the schedule due to the prolongations of certain operations. The recorded changes are given in Table 2, where $\Delta Q_{k}, \Delta S_{k}, k_{-}={ }_{-} 1, \ldots, 5$, and $\Delta \Phi$ denote the difference between the performance values, satisfaction grades, and the overall quality of the new and the base schedule respectively, Note that all the objectives have to be minimised and, therefore, the 
lower/higher values of the measures of performance $Q_{k}$ lead to the higher/lower values of the satisfaction grades $S_{k}$. Consequently, the positive (negative) values of $\Delta Q_{k}$ mean deterioration (improvement), respectively ${ }_{3}$ while the positive (negative) values of $\underline{\Delta S_{k}}$ and $\Delta \Phi$ denote improvement (deterioration), in the new schedule. For example, the maximum deterioration of the average weighted tardiness is 372 minutes, which leads to deterioration, 0.005 , of the satisfaction grade. On the other hand, the maximum improvement of the same criterion is 272 minutes, which gives improvement of 0.01 of the satisfaction grade.

Table 2. The range of changes of the performance values as a consequence of changing the upper bound of the processing time of each operation

\begin{tabular}{|c|l|l|l|l|}
\hline Performance & $\begin{array}{r}\text { Value in the base } \\
\text { schedule }\end{array}$ & \multicolumn{1}{|c|}{$\Delta Q_{k}$} & \multicolumn{1}{|c|}{$\Delta S_{k}$} & \\
\hline$Q_{1}$ & 789 (minutes) & {$[-272,372]$} & {$[-0.005,0.01]$} & \\
\hline$Q_{2}$ & 9 (jobs) & {$[-1,3]$} & {$[-0.095,0.031]$} & \\
\hline$Q_{3}$ & 722 (minutes) & {$[-17,16]$} & {$[-0.014,0.016]$} & \\
\hline$Q_{4}$ & 189370 (minutes) & {$[-2486,23001]$} & {$[-0.029,0.002]$} & \\
\hline$Q_{5}$ & 460602 (minutes) & {$[-7290,160692]$} & {$[-0.056,0.002]$} & \\
\hline$\Phi$ & 0.547 & & & {$[-0.022,0.007]$} \\
\hline
\end{tabular}

As an example, the job $J_{232}$ is identified as a critical job because the prolongation of the upper bound of $\mathrm{p}_{13}, 232$ by $90 \%$ of its modal time on machine $M_{13}$ leads to the worst overall deterioration of the schedule with the following effects on the performance measures: $\Delta Q_{1}=34, \Delta Q_{2}=3, \Delta Q_{3}=1, \Delta Q_{4}=1487, \Delta Q_{5}=15382, \Delta S_{1}$ $=-0.005, \Delta S_{2}=-0.095, \Delta S_{3}=-0.001, \Delta S_{4}=-0.001, \Delta S_{5}=-0.005$, while the overall quality of the schedule $\Phi$ is deteriorated by $0.022 \Delta \Phi=-0.022$.

4.3. Critical machines,

Deleted: Note that
Deleted: in the
Deleted: column
Deleted: . However, in
Deleted: the $\Delta S_{k}$ and $\Delta \Phi$ columns
Deleted: of the satisfaction grades and
the fitness function, respectively.

Deleted: J

Deleted: whose

Deleted: time

Deleted: Job $J_{232}$, Mmachine $M_{l 3}$ Iincrease of processing time $p_{13,232}$ by $90 \%$,Effects

Deleted: $. \Delta \Phi=-0.022 \pi$ Overall quality of the schedule is deteriorated by $0.022 \mathbb{I}$ II

Deleted: with respect to the performance values and the overall quality of the schedule 
Sensitivity analysis can help in identifying machines which are critical with respect to the performance values and the overall quality of the schedule. A numerical experiment is performed with the aim to select critical machines.

In this experiment the processing times of all the operations of each machine are increased in the same way as in the previous experiments. However, the processing times of all the operations to be processed on each machine are prolonged, one machine at the time, Table 3 presents the range of changes of the performance measures and the overall quality of the schedule caused by increasing the upper bounds of all the operations on each machine, one by one.

From this experiment we can select machines for which the prolongation of the processing times of jobs have the largest impact on the schedule performance. For example, data in Table 3 show that the most critical machine is machine $M_{6}$. Prolongations of the processing times of operations on this machine can reduce the overall quality of the schedule up to 0.55 on the satisfaction grade scale. On the other hand, the prolongation of the processing times of operations on machines $M_{3}, M_{9}$ and $M_{11}$ do not affect the overall performance of the schedule at all.

Such an analysis can help in evaluating the effect of running a certain machine at a lower speed, which will perhaps incur less cost. In addition, allocating one of a new or less experienced staff to operate a critical machine, which usually leads to the prolongation of the operation processing time, can be avoided.

4.4. Effects of prolongations of the operation processing times on the operation sequences

The sequence of jobs to be processed on a machine is determined by the dispatching rule associated with it. However, the prolongation of the processing times of operations on the machine may lead to a change of this sequence on the machine, and, also, to a change of the job sequences on some other machines. In the production environment in the printing industry, the sequence of jobs to be processed on each machine is important if setup operations are prepared in advance, based on the initial operation sequences on the machines determined by the base schedule. The change of the sequence may incur an additional cost.

Deleted: a

Deleted: a

Deleted: changed

Deleted: changing

Deleted: Changes

Deleted: changes

Deleted: or the effect of
Deleted: change

Deleted: environmentsin 
Table 3. Changes of performance values obtained by increasing the upper bound

Deleted: Range

of the processing times of all the operations on each machine

\begin{tabular}{|l|l|l|l|l|l|l|}
\hline Machine & \multicolumn{1}{|c|}{$\Delta Q_{1}$} & \multicolumn{1}{|c|}{$\Delta Q_{2}$} & \multicolumn{1}{c|}{$\Delta Q_{3}$} & \multicolumn{1}{|c|}{$\Delta Q_{4}$} & \multicolumn{1}{|c|}{$\Delta Q_{5}$} & \multicolumn{1}{|c|}{$\Delta \Phi$} \\
\hline$M_{1}$ & {$[-133,2863]$} & {$[0,24]$} & {$[-13,1]$} & {$[-1198,43514]$} & {$[4820,407478]$} & {$[-0.19,0]$} \\
\hline$M_{2}$ & {$[-109,1829]$} & {$[0,8]$} & {$[-4,9]$} & {$[-3032,23339]$} & {$[-3710,116385]$} & {$[-0.07,0]$} \\
\hline$M_{3}$ & {$[-3,58]$} & {$[0,0]$} & {$[0,3]$} & {$[0,1106]$} & {$[0,4583]$} & {$[0,0]$} \\
\hline$M_{4}$ & {$[-187,3]$} & {$[0,0]$} & {$[-7,3]$} & {$[-2251,0]$} & {$[-4377,1122]$} & {$[0,0.002]$} \\
\hline$M_{5}$ & {$[-257,2450]$} & {$[-1,17]$} & {$[-12,0]$} & {$[-3824,33656]$} & {$[-6605,164549]$} & {$[-0.13,0.008]$} \\
\hline$M_{6}$ & {$[-185,31106]$} & {$[-1,20]$} & {$[-12,9]$} & {$[-2297,42924]$} & {$[-45,5946]$} & {$[-0.55,0.007]$} \\
\hline$M_{7}$ & {$[-18,388]$} & {$[0,3]$} & {$[-4,12]$} & {$[-3013,8240]$} & {$[-1603,31643]$} & {$[-0.02,0]$} \\
\hline$M_{8}$ & {$[-148,121]$} & {$[0,0]$} & {$[-3,4]$} & {$[-3332,60]$} & {$[-5118,441]$} & {$[0,0.002]$} \\
\hline$M_{9}$ & {$[-9,38]$} & {$[0,0]$} & {$[-2,0]$} & {$[-529,813]$} & {$[-3451,2416]$} & {$[0,0]$} \\
\hline$M_{10}$ & {$[-48,57]$} & {$[0,0]$} & {$[-2,7]$} & {$[-103,1277]$} & {$[0,8374]$} & {$[-0.002,0]$} \\
\hline$M_{11}$ & {$[-3,5]$} & {$[0,0]$} & {$[0,1]$} & {$[-334,0]$} & {$[0,123]$} & {$[0,0]$} \\
\hline$M_{12}$ & {$[-6,182]$} & {$[0,1]$} & {$[-4,4]$} & {$[-489,1922]$} & {$[-9718,24]$} & {$[-0.007,0]$} \\
\hline$M_{13}$ & {$[-52,1006]$} & {$[1,6]$} & {$[-15,1]$} & {$[-139,3394]$} & {$[4607,49837]$} & {$[-0.04,0]$} \\
\hline$M_{14}$ & {$[-157,571]$} & {$[0,3]$} & {$[-13,13]$} & {$[-6038,19544]$} & {$[-6515,59754]$} & {$[-0.02,0.003]$} \\
\hline$M_{15}$ & {$[-11,422]$} & {$[0,2]$} & {$[-1,1]$} & {$[-207,8846]$} & {$[-76,4218]$} & {$[-0.01,0]$} \\
\hline$M_{16}$ & {$[-3,610]$} & {$[0,2]$} & {$[-4,6]$} & {$[-404,6377]$} & {$[0,51549]$} & {$[-0.02,0]$} \\
\hline$M_{17}$ & {$[-102,1729]$} & {$[0,9]$} & {$[-11,3]$} & {$[-879,25398]$} & {$[593,121464]$} & {$[-0.07,0]$} \\
\hline$M_{18}$ & {$[-3,117]$} & {$[0,1]$} & {$[-14,1]$} & {$[-977,348]$} & {$[-1869,10450]$} & {$[-0.004,0.002]$} \\
\hline
\end{tabular}


For every machine $i=1, \ldots, M$

$$
\begin{aligned}
& \text { For every job } j=1, \ldots, N \\
& S_{\text {before }}=\text { set of operations processed before operation }(i, j) \text { in the base schedule } \\
& S_{\text {after }}=\text { set of operations processed after operation }(i, j) \text { in the new schedule } \\
& S=S_{\text {before }} \cap \mathrm{S}_{\text {after }} \\
& N_{i j}=|S|, \quad(|| \text { denotes the cardinality of the set }) \\
& S D=\sum_{i}^{M} \sum_{j}^{N} N_{i j}
\end{aligned}
$$

Figure 7. Pseudo-code for the calculation of the sequence deviation 
Table 4. Effects of the prolongation of operation processing times of each machine on the operation sequence

\begin{tabular}{|c|c|c|c|c|}
\hline Machines & $\begin{array}{l}\text { Number of } \\
\text { operations }\end{array}$ & $\begin{array}{l}\text { Increase of the } \\
\text { operation time } \bar{\Phi} \overline{0}\end{array}$ & $\begin{array}{l}\text { Number of } \\
\text { affected machines }\end{array}$ & $\begin{array}{c}\text { Total sequence } \\
\text { deviation }\end{array}$ \\
\hline$M_{1}$ & 145 & 15 & 8 & 114 \\
\hline$M_{2}$ & 107 & 15 & 3 & 39 \\
\hline$M_{3}$ & 2 & $100^{v}$ & 4 & 9 \\
\hline$M_{4}$ & 33 & $100^{*}$ & 7 & 58 \\
\hline$M_{5}$ & 160 & 15 & 9 & 321 \\
\hline$M_{6}$ & 157 & 15 & 9 & 311 \\
\hline$M_{7}$ & 69 & 20 & 9 & 46 \\
\hline$M_{8}$ & 36 & 15 & 4 & 24 \\
\hline$M_{9}$ & 24 & 100 & 5 & 74 \\
\hline$M_{10}$ & 16 & $100^{\mathrm{s}}$ & 3 & 171 \\
\hline$M_{11}$ & 12 & 60 & 1 & 4 \\
\hline$M_{12}$ & 46 & 15 & 1 & 1 \\
\hline$M_{13}$ & 66 & 15 & 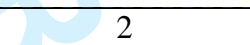 & 27 \\
\hline$M_{14}$ & 79 & 15 & 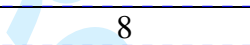 & 56 \\
\hline$M_{15}$ & 28 & 15 & 2 & 6 \\
\hline$M_{16}$ & 19 & 50 & - & 78 \\
\hline$M_{17}$ & 20 & 70 & 8 & 480 \\
\hline$M_{18}$ & 25 & 50 & 5 & 30 \\
\hline
\end{tabular}

We can observe that machines $M_{1}, M_{2}, M_{5}, M_{6}, M_{8}, M_{12}, M_{13}, M_{14}$, and $M_{15}$, are the most critical for the operation sequences in the sense that the increase of the processing time of their operations that causes the change of their sequences is small i.e. $15 \%$. Increase of the upper bound of the processing time of operations on machines $M_{5}, M_{6}$, and $M_{7}$ affected a large number of other machines, while machine $M_{17}$ had the largest sequence deviation.

Deleted: change Deleted: a

Deleted: Increment

\begin{tabular}{|l|}
\hline Deleted: $\%$ \\
\hline Deleted: $\%$ \\
\hline Deleted: $\%$ \\
\hline Deleted: $\%$ \\
\hline Deleted: $\%$ \\
\hline Deleted: $\%$ \\
\hline Deleted: $\%$ \\
\hline Deleted: $\%$ \\
\hline Deleted: $\%$ \\
\hline Deleted: $\%$ \\
\hline Deleted: $\%$ \\
\hline Deleted: $\%$ \\
\hline Deleted: $\%$ \\
\hline Deleted: $\%$ \\
\hline
\end{tabular}

Deleted: s

Deleted: the

Deleted: est

Deleted: the

Deleted: st 
Real world scheduling problems are often characterised by imprecise and uncertain job processing times As a consequence, in practice, it is not proper to consider processing times as deterministic values. It is much more appropriate to describe processing times by fuzzy numbers. Another important characteristic of the scheduling practice is that the quality of schedules depends on multiple measures of schedule performance. We identified five measures of performance: the average weighted tardiness, the number of tardy jobs, the total set-up time, the total idle time of machines and the total flow time of jobs. One approach to treat all the measures of performance simultaneously is the following one. First, the measures of performance are mapped linearly into the satisfaction grades, which take values from $[0,1]$ interval. Then all the satisfaction grades are aggregated and form a weighted sum which represents a single measure of the quality of a schedule.

The sensitivity analysis of the performance measures of a generated schedule with respect to the changes of job processing times helps in getting a better insight into the quality of schedules. The proposed numerical procedure for the sensitivity analysis is based on the prolongation of job processing times, job by job. It is done by widening the supports of fuzzy numbers which describe the processing times.

The sensitivity analysis enables the identification of critical jobs and critical machines in a schedule, in the sense that such jobs and such machines have the highest impact on the quality of a schedule. It is of great importance for scheduling practitioners and will contribute in bridging the gap that still exists between the productions scheduling theory and practice.

Our future work will include the investigation of how to make the generation of a base schedule robust to the prolongation of job processing times. Jensen (2003) developed an approach to robust scheduling that was motivated by the results obtained in the robust optimisation of continuous functions. It was shown that robust optima were located on broad peaks of the objective function. A robustness measure was defined which took into consideration all neighbour solutions of a schedule. The aim was to generate such a schedule with good quality neighbour solutions, so that when a disturbance, such as a machine breakdown, occurs one of them can work around it. We will investigate whether the similar idea is applicable to our problem. 


\section{Acknowledgments}

The authors would like to thank the Engineering and Physics Sciences Research Council (EPSRC), UK, for supporting this research (Grant No. GR/R95319/01 and GR/R95326/01). We would also like to acknowledge the support of our industrial collaborator Sherwood Press Ltd, Nottingham.

\section{References}

Blazewicz, J., Domschke, W. and Pesch, E., The job shop scheduling problem: Conventional and new solution techniques, European Journal of Operational Research- EJOR, 1996, 93, 1-33.

Dempster, M.A.H., Lenstra, J.K. and Rinnooy Kan, A.H.G., (Eds.), Deterministic and Stochastic Scheduling, 1982, (Reidel, Dordrecht).

Dubois, D., Prade, H. Operations on fuzzy numbers, International Journal of System Sciences, 1978, 9/6, 613-626.

Fargier, H., Fuzzy Scheduling: Principles and Experiments. In Fuzzy Information Engineering, A Guided Tour of Applications, edited by D. Dubois, H. Prade, and R.R. | Yager, pp. 655-668, 1996 (John Wiley \& Sons).

Fayad, C. and Petrovic, S., A Fuzzy Genetic Algorithm for Real-World Job-Shop Scheduling., In Innovations in Applied Artificial Intelligence, Lecture Notes in Artificial Intelligence 3533 edited by $\mathrm{M}$. Ali and $\mathrm{F}$. Esposito, pp. 524-533, 2005 (Springer).

Grabot, B. and Geneste, L., Dispatching rules in scheduling: a fuzzy approach, International Journal of Production Research, 1994, 32/4, 903-915.

Hall, N. and Posner, M., Sensitivity analysis for scheduling problems, Journal of Scheduling, 2004, 7/1, 49-83.

James, R.J.W. and Buchanan, J.T., Robustness of single machine scheduling problems to earliness and tardiness penalty errors, Annals of Operations Research, 1998, 76, 219-232.

Klir G. and Folger T., Fuzzy Sets, Uncertainty and Information, 1998 (Prentice Hall, New Jersey).

Kolen, A.W.J., Rinnooy Kan, A.H.G, van Hoesel C.P.M. and Wagelmans A.P.M, Sensitivity analysis of list scheduling heuristics, Discrete Applied Mathematics, 1994, $\mathbf{5 5} / \mathbf{2}, 145-162$
Deleted: In such complex production scheduling problems with uncertain processing times of jobs and multiple measures of schedule performance, it is important to make sensitivity analysis and evaluate the quality of a schedule by testing the consequences of changing the basic model parameters. We have used different experiments to numerically address some effects of changes of job processing times on all the measures of performance of a schedule. Numerical experimentation appears to be a unique practical way in getting trust in the quality of the produced schedule. The sensitivity analysis is used to identify the subsets of critical jobs and critical machines in the sense that such jobs and such machines mainly affect the quality of a schedule. The sensitivity analysis is very important in our attempts to generate the flexible and robust schedules and will contribute in bridging the gap that still exists between the productions scheduling theory and practice.

Deleted: pp

Formatted

Deleted: ,

Deleted: i

Deleted:

Deleted: 18th International Conference on Industrial and Engineering

Applications of Artificial Intelligence and

Expert Systems, IEA/AIE-2005, Bari, June 2005,

Deleted: 
Leung, J. (Eds) Handbook of Scheduling: Algorithms, Models, and Performance | Analysis , 2004, _Chapman \& Hall/CRCRC Press).

Petrovic, S. and Fayad, C., A Genetic Algorithm for Job shop Scheduling with Load Balancing, in AI 2005, Lecture Notes in Artificial Intelligence $3809^{\text {edited by S.Zhang }}$ and R.Jarvis, pp. 339-348, 2005_(Springer)

Petrovic, S., Fayad, C. and Petrovic, D., Job Shop Scheduling with Lot-sizing and Batching in an Uncertain Real-world Environment, in Proceedings of the 2nd Multidisciplinary International Conference on Scheduling: Theory and Applications (MISTA 2005), New York, USA, July 18-21, 2005, edited by G. Kendall, L. Lei and M.Pinedo, 2005, pp. 363-379.

Pinedo M., Scheduling: Theory, Algorithms, and Systems, Second Edition, 2002 (Prentice Hall, New Jersey)

Sakawa, M. and Kubota, R., Fuzzy Programming for Multiobjective Job Shop Scheduling with Fuzzy Processing Time and Fuzzy Duedate through Genetic Algorithms, European Journal of Operational Research - EJOR, 2000, 120/2, 393407.

Slowinski, R. and Hapke, M., (Eds.), Scheduling Under Fuzziness, 2000 (PhysicaVerlag).

Sotskov, Y.N., Stability of an Optimal Schedule, European Journal of Operational Research - EJOR, 1991, 55/1, 91-102.

Tseng, T.Y. and Klein, C., New Algorithm for the Ranking Procedure in Fuzzy Decision making, IEEE Transactions on Systems, Man and Cybernetics, 1989, 19/5, 1289-1296.

Watatani, Y., Fujii, S., A study on rescheduling policy in production systems, in the Proceedings of the JAPAN/USA Symposium on Flexible Automation, ASME, 2, 1992, pp. 1147-1150.
Deleted: ,

Deleted: 18th Australian Joint Conference on Artificial Intelligence, Sydney, Australia, December, 2005,

Deleted:

Deleted: -Verlag 